\title{
Los Derechos Humanos de la mujer peruana en el marco de protección del Derecho Internacional
}

Krúpskaya Ugarte Boluarte

Profesora de la Universidad de Alas Peruanas de las asignaturas de Derechos Humanos y Jurisdicción Internacional. Candidata a Doctora por la Universidad Carlos III de Madrid España. Egresada del Doctorado: Programa de Derechos Fundamentales. Con Diploma de Estudios Avanzados (DEA).

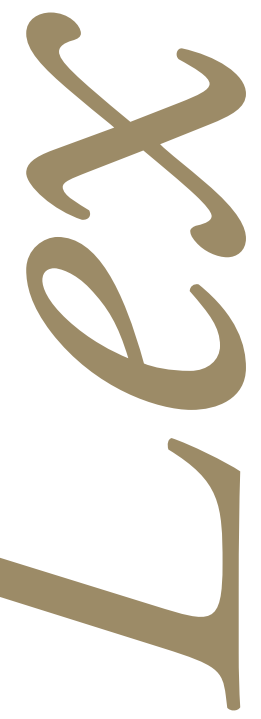




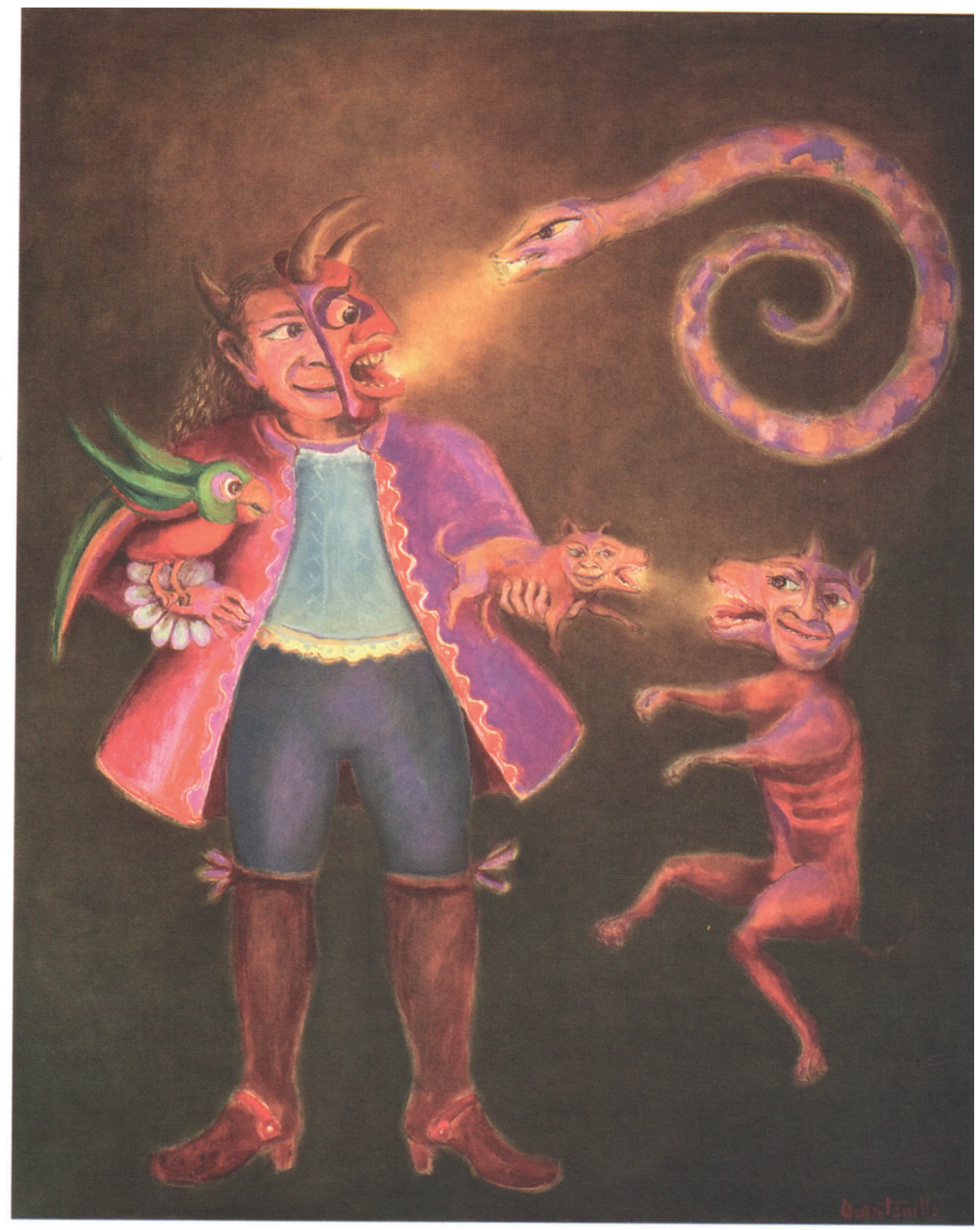

El disfrazado. Óleo, 2002 (107 cm. x 88 cm.) 


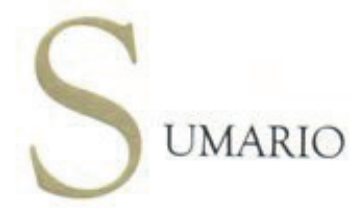

1.- Introducción; 2.- Un repaso histórico a la conquista de la Universalidad de los Derechos Humanos de las Mujeres. 3.- El Sistema Universal de Protección de los Derechos Humanos de la Mujer; 4.- Por qué es importante una Convención distinta y especial para la mujer en el marco de las Naciones Unidas. 5.- Conclusiones. 6.-Bibliografia.

\section{INTRODUCCIÓN}

El sistema de protección de los derechos humanos, tanto el Universal como el interamericano, se fue desarrollando en forma lenta y progresiva, como una conquista del individuo contra el Estado, que ha sido siempre el principal violador, lo que ha ido significando una limitación a su soberanía. En la actualidad existe una cantidad de instrumentos jurídicos cuyo contenido están dirigidos a proteger los derechos fundamentales de las personas, exigir la eficacia y vigor de los Estados en la aplicación de estos instrumentos ya sean estos del Sistema Universal o en el Sistema Interamericano. La Protección internacional de los derechos humanos ha sido el resultado de una lucha entre los detentadores de la soberania del Estado, por un lado y las que pretenden ampliar las prerrogativas del hombre, por otro lado; ampliando cada vez la posibilidad de llevar peticiones ante órganos internacionales, siendo estos logros, conquistas progresivas, nacionales e internacionales, con un reconocimiento y aplicación universal y efectivo. Originándose asi la llamada dimensión transnacional de protección de los derechos humanos y de la justicia, con la evidente intención del respeto de los derechos fundamentales a nivel internacional, a través de los organismos colocados fuera de las fronteras, esta realidad se concretó a través de la Declaración Universal, y los Pactos y Convenios sucesivos, en las diferentes materias. 
Esta defensa, se afianzo transnacionalmente, cobrando una importancia internacional, a través de Declaraciones, Pactos, Convenciones, en esta medida desarrollaré la implicancia universal y regional de la Convención sobre la eliminación de todas las formas de discriminación contra la mujer, y cuál es el real sentido de encaminar, de maximizar la importancia de los derechos contenidos en favor de las mujeres. En la actualidad, existen órganos de jerarquia internacional que pueden exigir a los Estados, la dación de preceptos juridicos que controlen la aplicación en el terreno interno del respeto y vigencia de los derechos del ser humano.

\section{INTRODUCCIÓN}

Plantear el tema, Los Derechos Humanos de la mujer peruana en el marco de protección del Derecho Internacional, en forma general; implica establecer que se debe tener en cuenta que la titularidad de los derechos se entienden en términos universales (considerando las peculiaridades diferenciales en el caso de mujeres y hombres). De modo, que si admitimos la universalidad de los derechos, no se podria hablar de derechos especificos de las mujeres, aunque esa especificidad tiene que salvarse necesariamente en las consecuencias prácticas que con lleva la aplicación y desarrollo del derecho.

Considero que la protección internacional puede entenderse en un doble sentido. Por una parte, se podria traducir en un análisis exegético de las disposiciones legales que de modo puntual han venido reconociendo para las mujeres una serie de derechos. Cuya titularidad habia sido negada para ellas. Y por otra se puede interpretar en el sentido de investigar el proceso llevado a cabo en los diferentes organismos internacionales respecto al reconocimiento de los derechos de la mujer.

La promoción y protección de los derechos de la mujer está muy relacionada con el tema de la discriminación de la mujer en el disfrute de los derechos humanos, es decir, mientras subsista la discriminación por género, las mujeres no podrán gozar plenamente de sus derechos humanos, y esta es una regla a nivel mundial. Por esta razón, la legislación internacional basa la protección de los derechos de la mujer fundamentalmente en el principio de no discriminación y en el principio de la igualdad entre hombres y mujeres.

En el caso peruano, ha adoptado importantes reformas legales para proteger los derechos de la mujer y para tratar de eliminar las formas de discriminación, subsistiendo sin embargo ciertas normas legales que implican discriminación. Existen avances en función a dos temas especificos, como es: la discriminación y la no violencia contra las mujeres. En relación a estos puntos de interés se han ido dando avances en el derecho interno de Perú, para fortalecer los Derechos de la Mujer peruana. 


\section{UN REPASO HISTÓRICO A LA CONQUISTA DE LA UNIVERSALIDAD DE LOS DERECHOS HUMANOS DE LAS MUJERES.}

La Carta de Naciones Unidas, firmada en junio de 1945, establece entre sus tres principales objetivos la defensa de los derechos y libertades, para todos los seres humanos sin distinción por razón de raza, sexo, lengua o religión. El preámbulo de la Carta, señala no sólo la fe en los derechos humanos fundamentales, en la dignidad de cada persona, sino también en la igualdad de derechos para mujeres y hombres.

El paso decisivo a la internacionalización definitiva de los derechos humanos en la Carta de 1945 cristaliza gracias a una serie de factores que concurrieron al final de la Segunda Guerra Mundial ...(como es ), el repudio contra los crímenes nazis, el deseo de paz, la afirmación de la supremacia de los valores democráticos, la re-fundación de la sociedad internacional organizada con la creación de la organización de las Naciones Unidas (ONU); [...] y sobre todo la voluntad decidida de los Estados fundadores de la ONU en conseguir un consenso politico que hiciera posible la construcción de un sistema universal de protección de los derechos humanos ${ }^{1}$.En este sentido, el punto culminante lo constituyó la Carta UN de 1945, (que) es el primer tratado internacional de alcance universal que afirma solemnemente la fe en los derechos fundamentales de todos los seres humanos, en la dignidad y el valor de la persona humana. Así pues, el proceso de internacionalización de los derechos humanos se ha incrementado notablemente desde la adopción de la Cara $\mathrm{UN}^{2}$.

Este avance de los derechos humanos (...) producirá un triple proceso de evolución: que es la positivización, la generalización y la internacionalización ${ }^{3}$. En ese sentido, la Declaración de Viena transó definitivamente la cuestión al afirmar, que la promoción y protección de todos los derechos humanos es una preocupación legitima de la Comunidad Internacional en el marco de los Propósitos y Principios establecidos en la Carta, en particular el Propósito de la cooperación internacional ${ }^{4}$.

Todo este desarrollo en protección y promoción de los "derechos humanos". Hace que este término (... sea) sin duda uno de los más usados en la cultura jurídica y politica actual, tanto por los científicos y los filósofos que se ocupan del hombre, del Estado y del Derecho, como

1 Villán Duran, Carlos, Curso de Derecho internacional de los derechos humanos, Madrid, Editorial TROTTA, 2002. 69 pp.

2 Villán Duran, Carlos, Curso de Derecho internacional de los derechos humanos, Madrid, Editorial TROTTA, 2002. $70.71 \mathrm{pp}$.

3 Peces-Barba Gregorio, Derecho Positivo de los Derechos Humanos. 13pp.

4 Villan Duran, Carlos, Op. Cit. 74 pp. 
por los ciudadanos ${ }^{5}$. También sea convertido en un término emotivo que suscita sentimientos entre sus destinatarios y respecto del cual la tentación de manipulación es permanente. ${ }^{6} \mathrm{En}$ tonces los derechos humanos (se conciben) como las facultades que el hombre se atribuye (...) inherentes a su condición, reflejadas en el consenso social obtenido a partir de la (...) voluntad de los individuos. ${ }^{?}$

Es importante, resaltar que los derechos fundamentales son un concepto histórico del mundo moderno que surge progresivamente a partir del tránsito a la modernidad. Peces-Barba ${ }^{8}$ señala, "que la idea central, que podemos encontrar en todo momento histórico, será la de la dignidad humana.

Los derechos humanos emergen [y erosionan] como principio constitucional del DI. Pero no "desplaza[n]" el viejo principio, también constitucional, de la soberanía de los Estados. Por lo que ambos principios constitucionales coexisten y se interrelacionan en una tensión dialéctica que debe tenerse muy presente para comprender las tensiones intrinsecas del DIDH en la fase actual de su evolución histórica?.

El Consejo Económico y Social, [es] uno de los seis principales órganos de Naciones Unidas; [su] ... principal Comisión fue la de Derechos Humanos (...) en la sesión inaugural de Naciones Unidas celebrada en Londres en 1946, la Sra. Eleanor Roosevelt, que formaba parte de la delegación americana, leyó una carta abierta a todas las mujeres del mundo en la que animaba a la participación de más mujeres en el trabajo de Naciones Unidas y, al tiempo, alentaba a los Gobiernos a tener en cuenta la causa de las mujeres.

Después de algunos debates, el Consejo concluyó que necesitaba un asesoramiento especial para problemas relacionados con la situación de las mujeres, y que era necesaria la creación de un órgano específico ${ }^{10}$. En febrero de 1946, el Consejo votó la creación de la Subcomisión sobre la situación de las mujeres. Cuatro meses más tarde, la Subcomisión desapareció para ser sustituida por la Comisión sobre la situación de las mujeres. Désde entonces, podria afirmarse que Naciones Unidas, a través de la Comisión, ha llevado a cabo una doble actuación, por la

5 Gregorio Peces-Barba Martinez. "Curso de Derechos Fundamentales" Teoria General. Universidad Carlos III de Madrid. Boletin Oficial del Estado. Madrid, 1995. 21 pp.

6 Ibid.

7 Ignacio Ara PINILLA, Hacia una Definición Explicativa de los Derechos Humanos, DERECHOS Y LIBERTADES, Revista del Instituto Bartolomé de las Casas. Universidad Carlos III de Madrid, 1993 107pp.

8 Peces - Barba Gregorio, Op.Cit. 11pp.

9 Carrillo Salcedo J.A, Soberania de los Estados y derechos humanos en el Derecho internacional contemporáneo. Cit. 131pp.

10 Ibid. 
via política y por la juridica. Entonces, resumiendo, la Declaración Universal (...) [es] "un ideal común por el que todos los pueblos y naciones deben esforzarse" ${ }^{11}$ (...) [y] por que es, el primer documento relativo a los derechos humanos elaborado por una Organización Universal ${ }^{12}$.

Sus principios han sido recogidos en las Constituciones y ordenamientos juridicos de numerosos Estados, y un gran número de tratados de derechos humanos se refieren a ella, confirmando su innegable relevancia moral, política y juridica (...) la Declaración por su carácter moral y la importancia juridica y política que ha adquirido con el transcurso del tiempo, constituye un hito en la lucha de la Humanidad por la libertad y la dignidad, a la altura de la carta magna inglesa, de la Declaración de Independencia americana y de la Declaración francesa de los Derechos del Hombre y del Ciudadano ${ }^{13}$. La Declaración Universal (...) supuso "una victoria (no total, ciertamente) de la humanidad entera"14.

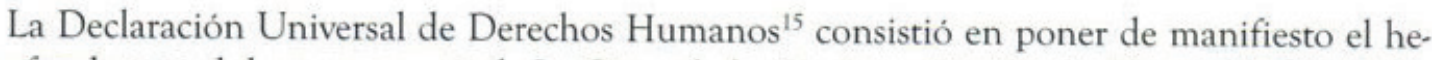
cho fundamental de que, a partir de La Carta de las Naciones Unidas, la Comunidad Internacional experimentó una auténtica revolución juridica: reconocer los derechos fundamentales de la persona, universales e indivisibles, como uno de sus intereses fundamentales y uno de los principios constitucionales del orden internacional ${ }^{16}$. A partir de este punto, nos interesa ver el desenlace de esta norma juridica universal, y ver el avance y la transformación que sufren los derechos humanos de la mujer.

\section{EL SISTEMA UNIVERSAL DE PROTECCIÓN DE LOS DERECHOS HUMA- NOS DE LA MUJER}

El Preámbulo de la Declaración Universal parte de la idea de que los derechos humanos tienen su raiz en la dignidad y el valor de la persona humana (apartados $1^{\circ} \mathrm{Y} 5^{\circ}$, confirmados por el artículo 1). Por eso corresponde a todos los miembros de la familia humana derechos iguales e inalienables (apartado $1^{\circ}$, confirmado por el art.2).

11 Carrillo Salcedo, Juan Antonio, La declaración Universal de Derechos Humanos, cincuenta años después. Minima Trotta. Madrid, 1999. 18 pp.

12 Carrillo Salcedo, Juan Antonio, Soberania de los estados y derechos humanos en derecho internacional contemporáneo. Segunda Edición. Tecnos. Madrid 2001. 49 pp.

13 Carrillo. JA. Op Cit. 18 pp.

14 Cassese Antonio, Los derechos humanos en el mundo contemporáneo, Ariel, Barcelona 1991, 53 pp.

15 René Cassin tuvo el acierto de sugerir, y su propuesta unánimemente aceptada, que el título de la declaración no fuese el de "Declaración Internacional", sino el de "Declaración Universal de Derechos Humanos". Con relación a los derechos económicos y sociales, la Declaración Universal expresa igualmente un consenso entre los bloques ideológicos enfrentados, enunció derechos civiles y politicos e incluyó también derechos económicos y sociales.

16 Carrillo Salcedo, Juan Antonio.: La Declaración Universal de Derechos Humanos, cincuenta años después. Minima Trotta. Madrid, 1999. 18 pp. 
Resulta asi que los seres humanos están divididos, en una especie de apartheid global, en dos grandes sectores: el de aquellos para quienes los derechos humanos son una realidad cotidiana. Y el de aquellos otros para quienes son todavia un objeto por el que luchar ... (es el caso de) los derechos de la mujer y de los pueblos indigenas, por ejemplo, (que) no han recibido un apoyo adecuado; los derechos económicos, sociales y culturales han sido retóricamente ensalzados, pero nunca abordados real y resueltamente en las Naciones Unidas ${ }^{17}$.

Ahora, la gran importancia, de la Declaración Universal de los Derechos Humanos, ha servido (...) de fundamento a los esfuerzos de la Organización de las Naciones Unidas y de otras Organizaciones Internacionales para su protección Internacional, como es el caso de las mujeres. Frente a esta necesidad, la Asamblea General de las Naciones Unidas, el 18 de diciembre de 1979, ... culmina; la Convención sobre la Eliminación de todas las Formas de Discriminación contra la Mujer ...encargado de desarrollar el mandato establecido en el preámbulo de la Carta de las Naciones Unidas de que esta organización tome medidas para mejorar la condición de las mujeres, ya que "los pueblos de las Naciones Unidas están resueltos a reafirmar la fe en los derechos fundamentales del hombre, en la dignidad y el valor de la persona humana y en la igualdad de derechos de hombres y mujeres y de las naciones grandes y pequeñas" ${ }^{18}$.

La Convención fue, pues, un paso decisivo en el proceso de desarrollo de los derechos de la mujer, impulsado por la Comisión de la Condición Juridica y Social de la mujer a través de diversas actuaciones, algunas dirigidas a concienciar a la opinión pública de los Estados sobre la necesidad de mejorar la condición de las mujeres para promover los derechos humanos y la paz, y otras conducentes a la aprobación de una serie de instrumentos jurídicos como: La Convención sobre los Derechos Politicos de la Mujer (1952), La Convención sobre Nacionalidad de la Mujer Casada (1957), La Convención sobre el Consentimiento para el Matrimonio. La Edad Minima para Contraer Matrimonio y Registro de Matrimonios (1962).

Durante el Decenio se examinaron en dos ocasiones (Copenhague en 1980 y en Nairobi en 1985) los progresos realizados y los obstáculos encontrados en él desarrollo del Plan de Acción Mundial. La Década sirvió para avanzar considerablemente en reformas legales a favor de la igualdad juridica entre mujeres y hombres ${ }^{19}$.

17 Carrillo Salcedo Juan Antonio, La Declaración Universal de Derechos Humanos, cincuenta años después. Minima Trotta. Madrid, 1999. 23 pp

18 Bustelo Garcia Del. Real, Carlota.: La Convención sobre la eliminación de todas las formas de discriminación contra la mujer". Garantia internacional de los derechos sociales. contribución de Naciones Unidas al progreso y desarrollo social, los derechos del niño, la eliminación de la discriminación de la mujer y desarrollo del Voluntariado. Ministerio de Asuntos Sociales. Madrid 1990. 211.pp.

19 El desarrollo económico y cultural aporta a las mujeres, en especial a las mujeres más pobres, los medios necesarios para poder reivindicar, obtener, dısíntar y utilizar la igualdad de oportunidad. Por otro lado la paz, 
La elaboración y aprobación de la Convención sobre la Eliminación de todas las formas de Discriminación contra la mujer se inserta, pues, en este conjunto de actuaciones y es uno de los logros más importantes del Decenio de la Mujer de las Naciones Unidas ya que representa la Carta Magna de los derechos humanos de la mujer y, como tal, constituye un instrumento fundamental para avanzar en la no discriminación por razón de sexo. El texto del 79 ha sido reforzado con la aprobación del Protocolo opcional al Convenio, adoptado por la Asamblea en diciembre de 1999. Ambos texto suponen el reconocimiento juridico de las acciones positivas.

Aunque la igualdad entre mujeres y hombres no es plena en muchos lugares del mundo, y aunque incluso en algunas sociedades en las que legalmente está reconocida la igualdad, ésta no es real, puede afirmarse que el trabajo de Naciones Unidas ha fomentado, entre otras cosas, un cambio sustancial. Y éste ha sido el de conseguir que la igualdad de las mujeres haya pasado de ser un problema exclusivo de las mujeres a convertirse en un problema social que requiere necesariamente la colaboración de los hombres.

\section{POR QUÉ ES IMPORTANTE UNA CONVENCIÓN DISTINTA Y ESPECIAL PARA LA MUJER EN EL MARCO DE LAS NACIONES UNIDAS.}

La Carta Internacional de Derechos Humanos establece un amplio conjunto de derechos que se aplican a todas las personas, incluidas las mujeres. Valdria preguntarnos ¿Por qué entonces fue necesario adoptar un instrumento juridico especial para la mujer?, se consideró necesario contar con otros medios para proteger los derechos humanos de la mujer porque el mero hecho de su "humanidad" no ha sido suficiente para garantizar a las mujeres la protección de sus derechos. En el preámbulo de la Convención sobre la eliminación de todas las formas de discriminación contra la mujer se explica que, a pesar de la existencia de otros instrumentos, las mujeres siguen sin tener derechos iguales a los de los hombres. La discriminación contra la mujer sigue existiendo en todas las sociedades.

La Convención fue aprobada por la Asamblea General en 1979 para reforzar las disposiciones de los instrumentos internacionales existentes destinadas a combatir la persistencia de la discriminación contra la mujer (y) para combatir la discriminación por motivos de sexo, la Convención requiere a los Estados Partes que reconozcan la importante contribución económica y social de la mujer a la familia y la sociedad en su conjunto. Hace hincapié en que la discriminación obstaculiza el crecimiento económico y la prosperidad ${ }^{20}$. Otra caracteristica importante

aunque imprescindible para mejorar la situación de las mujeres, no garantiza la igualdad a la mujer ni siquiera la ausencia de violencia hacia ella. Los informes y estudios realizados durante la década sacaron a la luz, ante la opinión pública mundial, las agresiones que sufren en todos los paises las mujeres por parte de los hombres en el ámbito familiar, en la calle y en el centro de trabajo.

20. Folleto Informativo No 22. Discriminación Contra la Mujer: La Convención y el Comité. 
de la Convención es el reconocimiento explicito de la meta de una igualdad real, además de juridica, y de la necesidad de medidas especiales de carácter temporal para alcanzarla.

La Convención parte de la constatación como circunstancia fundamental a tener en cuenta, de la condición oprimida de un grupo humano debido a una característica biológica que es el sexo y del reconocimiento de que la desigualdad real entre hombres y mujeres es un hecho lamentable en todas las sociedades del mundo ${ }^{21}$. Todo el contenido de la Convención gira alrededor de dos conceptos: La igualdad entre los sexos, como postulado básico, y la eliminación de la discriminación contra la mujer en sus distintas formas, como objetivo a alcanzar.

La Convención sobre toda forma de discriminación contra la mujer (aprobada en 1979, abierta a la firma desde 1980 y en vigor desde 1981), fue ratificada por Perú en 1982, y define la discriminación contra la mujer como: “...toda distinción, exclusión o restricción basada en el sexo, que tenga por objeto o por resultado menoscabar o anular el reconocimiento, goce o ejercicio por la mujer, independientemente de su estado civil, sobre la base de la igualdad del hombre y la mujer, de los derechos humanos y las libertades fundamentales...". Y agrega en su articulo 2 que: "Los Estados Partes condenan la discriminación contra la mujer en todas sus formas y convienen en seguir, por todos los medios apropiados y sin dilaciones, una política encaminada a eliminar la discriminación contra la mujer".

El Estado Peruano suscribió la Convención sobre la Eliminación de Todas las Formas de Discriminación contra la Mujer, y la ratificó el 13 de septiembre de 1982. Dentro de este marco se ha elaborado el Proyecto del Protocolo Facultativo de la Convención sobre la Eliminación de todas las formas de Discriminación contra la Mujer, en junio de 1998. Este instrumento proyecta básicamente el derecho a presentar denuncias individuales ante el Comité para la Eliminación de la discriminación contra la Violación a la Convención y la facultad del Comité de solicitar informes a los Estados Partes. Asimismo, en Julio de 1994 se suscribió la Convención Interamericana para Prevenir, Sancionar y Erradicar la Violencia contra la Mujer, la misma que fue ratificada el 4 de junio de 1996.

\section{CONCLUSIONES}

A manera de conclusión podemos señalar, que la situación de las mujeres ha mejorado de una manera sustancial a nivel internacional en algunos paises, pero falta mucho por reforzar

BUSTELO GARCIA DEL REAL, Carlota, La Convención sobre la eliminación de todas las formas de discriminación contra la mujer". Garantia internacional de los derechos sociales.- contribución de Naciones Unidas al progreso y desarrollo social, los derechos del niño, la eliminación de la discriminación de la mujer y desarrollo del Voluntariado. Ministerio de Asuntos Sociales. Madrid 1990. 214.pp 
en paises Iberoamericanos, como es el caso de Perú. La presencia de mujeres en la vida pública ha experimentado un importante avance, que no sólo se refiere a un mayor número de representantes femeninas en parlamentos, municipios y gobiernos, sino que también incide en una mayor presencia de mujeres en puestos y cargos de decisión. Del mismo modo, se ha seguido acelerando la incorporación de mujeres a otros espacios públicos, como asociaciones de mujeres $\mathrm{u}$ organizaciones no gubernamentales.

Todo esto nos lleva a confirmar que el proceso de cambio que se empezó a configurar con fuerza, en la década pasada, ha ayudado a consolidar y ampliar sus efectos, rompiendo el carácter androcéntrico que ha caracterizado a los espacios públicos. Si en la política se aprecian avances lentos pero sostenidos como claramente se constata en la presencia de mujeres en el Parlamento, es el caso de las mujeres peruanas en el Parlamento Peruano. El hecho social de suponer que las mujeres son un colectivo poco emprendedor, parece debilitarse, al constatarse, en este quinquenio, un crecimiento sostenido de mujeres empresarias y empleadoras.

Es importante, el nivel del compromiso de los Estados, que al aceptar la Convención, los Estados y Gobiernos se comprometan a asumir una serie de medidas para erradicar la discriminación contra de las mujeres en todas sus formas.

Ahora, es necesario dejar en claro, que las disposiciones de la CEDAW tienen fuerza de ley, por lo que resulta ser el compromiso internacional vinculante de mayor impacto en la redefinición de las condiciones de vida y posición de las mujeres desde la década del 80 . Su rol como marco internacional para la planificación de políticas públicas y acciones de desarrollo la hace de plena vigencia y actualidad. Los avances politicos y de los planes y programas de los Estados, dirigidos a la promoción de la mujer están vinculados a lo establecido en la CEDAW pues son un reflejo interno de la agenda planteada por esta Convención.

\section{BIBLIOGRAFÍA}

ARA PINILLA, Ignacio, Hacia una Definición Explicativa de los Derechos Humanos, DERECHOS Y LIBERTADES, Revista del Instituto Bartolomé de las Casas. Universidad Carlos III de Madrid, 1993.

BUSTELO GARCÍA DEL REAL, Carlota, La Convención sobre la eliminación de todas las formas de discriminación contra la mujer". Garantía internacional de los derechos sociales. contribución de Naciones Unidas al progreso y desarrollo social, los derechos del niño, la eliminación de la discriminación de la mujer y desarrollo del Voluntariado. Ministerio de Asuntos Sociales. Madrid 1990. 
CARRILlO SALCEDO, Juan Antonio, La Declaración Universal de Derechos Humanos, cincuenta años después. Mínima Trotta. Madrid, 1999.

- Soberanía de los estados y derechos humanos en derecho internacional contemporáneo. Segunda Edición. Tecnos. Madrid 2001.

CASSESE Antonio, Los derechos humanos en el mundo contemporáneo, Ariel, Barcelona 1991.

- Derecho Positivo de los Derechos Humanos.

DE CASTRO CID, Benito. : "El Reconocimiento de los Derechos Humanos" Editorial Tecnos, S.A. Madrid 1982.

DURAN Paloma. La protección internacional del ejercicio de los derechos humanos por las mujeres- Igualdad de mujeres y hombres a la luz del Tratado de Ámsterdam. Instituto de la mujer. Madrid. Debate № 31 .

Folleto Informativo № 22.Discriminación Contra la Mujer: La Convención y el Comité.

O DONNELL, Daniel: Protección Internacional de los Derechos Humanos Comisión Andina de Juristas. Fundación Friedrich Naumann. 2da. Edición 1989. Lima Perú.

PECES BARBA Gregorio. Iñiguez de Onzoño, Liborio Hierro Santiago. Llamas Angel, Derecho Positivo de los Derechos Humanos, Editorial Debate, Primera Edición: abril 1987. Madrid.

PECES- BARBA MARTINEZ, Gregorio. De Asis Roig, Rafael. Fernandez Liesa, Carlos R. Llamas Cascón, Ángel. : “Curso de Derechos Fundamentales Teoria General”. Universidad Carlos III de Madrid. Boletin Oficial del Estado, Madrid, 1995.

PECES-BARBA MARTÍNEZ Gregorio. "Curso de Derechos Fundamentales" Teoría General. Universidad Carlos III de Madrid. Boletin Oficial del Estado. Madrid, 1995.

- "Concepto de Derechos Humanos y Problemas Actuales. Derechos y Libertades, Revista del Instituto Bartolomé de las Casas, Universidad Carlos III de Madrid, Boletin Oficial del Estado. Año I febrero-octubre 1993 numero 1.

NIKKEN, Pedro, La Protección Internacional de los Derechos Humanos: Su Desarrollo Progresivo", Instituto Interamericano de Derechos Humanos Editorial Civitas, S.A., Primera Edición.1987. Madrid España. 
TRUYOL Y SERRA, Antonio. : "Los Derechos Humanos, Declaraciones y Convenios Internacionales”, Nueva edición actualizada, con una nota adicional: La Evolución Juridico-Positiva Última y una Cronologia relativa a los derechos humanos., Editorial Tecnos S.A. 1984, 1ra reimpresión 1984. Madrid España.

VILLÁN DURAN, Carlos, Curso de Derecho internacional de los derechos humanos, Madrid, Editorial TROTTA, 2002. 
